\title{
OPEN INTERSECTION NUMBERS AND THE WAVE FUNCTION OF THE KDV HIERARCHY
}

\author{
A. BURYAK
}

\begin{abstract}
Recently R. Pandharipande, J. Solomon and R. Tessler initiated a study of the intersection theory on the moduli space of Riemann surfaces with boundary. They conjectured that the generating series of the intersection numbers is a specific solution of a system of PDEs, that they called the open $\mathrm{KdV}$ equations. In this paper we show that the open $\mathrm{KdV}$ equations are closely related to the equations for the wave function of the KdV hierarchy. This allows us to give an explicit formula for the specific solution in terms of Witten's generating series of the intersection numbers on the moduli space of stable curves.
\end{abstract}

\section{INTRODUCTION}

Denote by $\mathcal{M}_{g, n}$ the moduli space of smooth complex algebraic curves of genus $g$ with $n$ distinct marked points. In [DM69] P. Deligne and D. Mumford defined a natural compactification $\mathcal{M}_{g, n} \subset \overline{\mathcal{M}}_{g, n}$ via stable curves (with possible nodal singularities). The moduli space $\overline{\mathcal{M}}_{g, n}$ is a nonsingular complex orbifold of dimension $3 g-3+n$.

A new direction in the study of the moduli space $\overline{\mathcal{M}}_{g, n}$ was opened by E. Witten [Wit91]. The class $\psi_{i} \in H^{2}\left(\overline{\mathcal{M}}_{g, n} ; \mathbb{C}\right)$ is defined as the first Chern class of the line bundle over $\overline{\mathcal{M}}_{g, n}$ formed by the cotangent lines at the $i$-th marked point. Intersection numbers $\left\langle\tau_{k_{1}} \tau_{k_{2}} \ldots \tau_{k_{n}}\right\rangle_{g}^{c}$ are defined as follows:

$$
\left\langle\tau_{k_{1}} \tau_{k_{2}} \ldots \tau_{k_{n}}\right\rangle_{g}^{c}:=\int_{\overline{\mathcal{M}}_{g, n}} \psi_{1}^{k_{1}} \psi_{2}^{k_{2}} \ldots \psi_{n}^{k_{n}} .
$$

The superscript $c$ here signals integration over the moduli of closed Riemann surfaces. Let us introduce variables $u, t_{0}, t_{1}, t_{2}, \ldots$ and consider the generating series

$$
F^{c}\left(t_{0}, t_{1}, \ldots ; u\right):=\sum_{\substack{g \geq 0, n \geq 1 \\ 2 g-2+n>0}} \frac{u^{2 g-2}}{n !} \sum_{k_{1}, \ldots, k_{n} \geq 0}\left\langle\tau_{k_{1}} \tau_{k_{2}} \ldots \tau_{k_{n}}\right\rangle_{g}^{c} t_{k_{1}} t_{k_{2}} \ldots t_{k_{n}} .
$$

E. Witten ([Wit91] $)$ conjectured that the exponent $\exp \left(F^{c}\right)$ is a tau-function of the KdV hierarchy. Witten's conjecture was proved by M. Kontsevich ([Kon92]). There was a later reformulation of Witten's conjecture due to R. Dijkgraaf, E. Verlinde and H. Verlinde ([DVV91]). They defined certain quadratic differential operators $L_{n}, n \geq-1$, and proved that Witten's conjecture is equivalent to the equations $L_{n} \exp \left(F^{c}\right)=0$, that are called the Virasoro equations.

In PST14 the authors initiated a study of the intersection theory on the moduli space of Riemann surfaces with boundary. They introduced intersection numbers on this moduli space and called them the open intersection numbers. The authors completely described them in genus 0. In higher genera they conjectured that the generating series of the open intersection numbers satisfies certain partial differential equations that are analogous to the $\mathrm{KdV}$ and the Virasoro equations. In [PST14] these equations were called the open KdV and the open Virasoro equations.

In Bur14 we studied the open KdV equations in detail. First, we proved that the system of the open $\mathrm{KdV}$ equations together with the initial condition, that corresponds to the simplest open intersection numbers in genus 0 , has a unique solution. We call this solution the open potential $F^{o}$. Second, in Bur14 we showed that the open potential $F^{o}$ satisfies the open

2010 Mathematics Subject Classification. Primary 35Q53; Secondary 14H10.

Key words and phrases. Riemann surfaces with boundary, moduli space, KdV equations. 
Virasoro equations. Therefore we proved that the open KdV and the open Virasoro equations give equivalent descriptions of the intersection numbers on the moduli space of Riemann surfaces with boundary. This was left in [PST14 as a conjecture.

In this paper we show that the system of the open $\mathrm{KdV}$ equations is closely related to the system of equations for the wave function of the KdV hierarchy. This allows us to give an explicit formula for the open potential $F^{o}$ in terms of the Witten potential $F^{c}$.

In [PST14] the authors didn't consider the contangent line bundles at boundary marked points, so the generating series of the open intersection numbers depends only on the variables $t_{0}, t_{1}, \ldots$ and $s$. In [Bur14] we suggested a natural way to introduce new variables $s_{1}, s_{2}, \ldots$ in the open potential $F^{o}$. The resulting power series is called the extended open potential. The new variables $s_{1}, s_{2}, \ldots$ can be viewed as descendants of boundary points. Actually, in this paper we derive an explicit formula for the extended open potential. We also construct Virasoro type equations for the extended open potential. Surprisingly, our new Virasoro equations are even simpler then the open Virasoro equations for the potential $F^{o}$.

1.1. Moduli of Riemann surfaces with boundary. Here we briefly recall the main definitions from [PST14].

Let $\Delta \in \mathbb{C}$ be the open unit disk, and let $\bar{\Delta}$ be its closure. An extendable embedding of the open disk $\Delta$ in a compact Riemann surface $f: \Delta \rightarrow C$ is a holomorphic map which can be extended to a holomorphic embedding of an open neighborhood of $\bar{\Delta}$. Two extendable embeddings are disjoint, if the images of $\bar{\Delta}$ are disjoint.

A Riemann surface with boundary $(X, \partial X)$ is obtained by removing a finite positive number of disjoint extendable open disks from a connected compact Riemann surface. A compact Riemann surface is not viewed here as Riemann surface with boundary.

To a Riemann surface with boundary $(X, \partial X)$, we can canonically construct the double via the Schwartz reflection through the boundary. The double $D(X, \partial X)$ of $(X, \partial X)$ is a compact Riemann surface. The doubled genus of $(X, \partial X)$ is defined to be the usual genus of $D(X, \partial X)$.

On a Riemann surface with boundary $(X, \partial X)$, we consider two types of marked points. The markings of interior type are points of $X \backslash \partial X$. The markings of boundary type are points of $\partial X$. Let $\mathcal{M}_{g, k, l}$ denote the moduli space of Riemann surfaces with boundary of doubled genus $g$ with $k$ distinct boundary markings and $l$ distinct interior markings. The moduli space $\mathcal{M}_{g, k, l}$ is defined to be empty unless the stability condition

$$
2 g-2+k+2 l>0
$$

is satisfied. The moduli space $\mathcal{M}_{g, k, l}$ is a real orbifold of real dimension $3 g-3+k+2 l$.

The cotangent line classes $\psi_{i} \in H^{2}\left(\mathcal{M}_{g, k, l} ; \mathbb{C}\right)$ are defined (as before) as the first Chern classes of the cotangent line bundles associated to the interior markings. In [PST14], cotangent lines at the boundary points are not considered. Open intersection numbers are defined by

$$
\left\langle\tau_{a_{1}} \tau_{a_{2}} \ldots \tau_{a_{l}} \sigma^{k}\right\rangle_{g}^{o}:=\int_{\overline{\mathcal{M}}_{g, k, l}} \psi_{1}^{a_{1}} \psi_{2}^{a_{2}} \ldots \psi_{l}^{a_{l}} .
$$

To rigorously define the right-hand side of (1.1), at least three significant steps must be taken:

- A natural compactification $\mathcal{M}_{g, k, l} \subset \overline{\mathcal{M}}_{g, k, l}$ must be constructed. Candidates for $\overline{\mathcal{M}}_{g, k, l}$ are themselves real orbifolds with boundary $\partial \overline{\mathcal{M}}_{g, k, l}$;

- For integration over $\overline{\mathcal{M}}_{g, k, l}$ to be well-defined, boundary conditions of the integrand along $\partial \overline{\mathcal{M}}_{g, k, l}$ must be specified;

- Orientation issues should be resolved, since the moduli space $\mathcal{M}_{g, k, l}$ is in general nonorientable.

All three steps are completed in genus 0 in [PST14]. The higher genus constructions will appear in upcoming work of J. P. Solomon and R. J. Tessler [ST]. 
1.2. Open $\mathbf{K d V}$ equations. Here we recall the open $\mathrm{KdV}$ equations and formulate the main conjecture from [PST14.

Let $F$ be a formal power series in the variables $t_{0}, t_{1}, \ldots$ and $s$ with the coefficients from $\mathbb{C}\left[u, u^{-1}\right]$. The open $\mathrm{KdV}$ equations are the following partial differential equations:

$$
\frac{2 n+1}{2} \frac{\partial F}{\partial t_{n}}=u \frac{\partial F}{\partial s} \frac{\partial F}{\partial t_{n-1}}+u \frac{\partial^{2} F}{\partial s \partial t_{n-1}}+\frac{u^{2}}{2} \frac{\partial F}{\partial t_{0}} \frac{\partial^{2} F^{c}}{\partial t_{0} \partial t_{n-1}}-\frac{u^{2}}{4} \frac{\partial^{3} F^{c}}{\partial t_{0}^{2} \partial t_{n-1}}, \quad n \geq 1 .
$$

In [PST14] the authors conjectured, that the generating series

$$
F^{o, \text { geom }}\left(t_{0}, t_{1}, \ldots, s ; u\right):=\sum_{\substack{g, k, l \geq 0 \\ 2 g-2+k+2 l>0}} \sum_{a_{1}, \ldots, a_{l} \geq 0} \frac{u^{g-1}}{k ! l !}\left\langle\tau_{a_{1}} \tau_{a_{2}} \ldots \tau_{a_{l}} \sigma^{k}\right\rangle_{g}^{o} s^{k} \prod_{i=1}^{l} t_{a_{i}}
$$

satisfies the open KdV equations. The following initial condition follows easily from the definitions:

$$
\left.F^{o, g e o m}\right|_{t_{\geq 1}=0}=u^{-1}\left(\frac{s^{3}}{6}+t_{0} s\right) .
$$

It is clear that the open $\mathrm{KdV}$ equations together with this initial condition and the closed potential $F^{c}$ completely determine the power series $F^{o, g e o m}$. Denote by $F^{o}$ a unique solution of the system (1.2), that satisfies the initial condition (1.3). The existence of such a solution is non-trivial and was proved in [Bur14]. The power series $F^{o}$ will be called the open potential. So the main conjecture from [PST14] can be formulated as the equation $F^{o, g e o m}=F^{o}$.

In Bur14] we proved, that the power series $F^{o}$ satisfies also the following equation:

$$
\frac{\partial F^{o}}{\partial s}=u\left(\frac{1}{2}\left(\frac{\partial F^{o}}{\partial t_{0}}\right)^{2}+\frac{1}{2} \frac{\partial^{2} F^{o}}{\partial t_{0}^{2}}+\frac{\partial^{2} F^{c}}{\partial t_{0}^{2}}\right) .
$$

This equation allows us to eliminate the derivatives by $s$ on the right-hand side of (1.2). Therefore the potential $F^{o}$ has the following equivalent description. It is a unique solution of the system

$$
\frac{2 n+1}{2 u^{2}} F_{n}=\left(\frac{1}{2} \frac{\partial^{2}}{\partial t_{0}^{2}}+F_{0} \frac{\partial}{\partial t_{0}}+\frac{1}{2} F_{0}^{2}+\frac{1}{2} F_{0,0}+F_{0,0}^{c}\right) F_{n-1}+\frac{1}{2} F_{0} F_{0, n-1}^{c}+\frac{3}{4} F_{0,0, n-1}^{c}, n \geq 1,
$$

with the initial condition

$$
\left.F\right|_{\substack{t_{1}=0 \\ s=0}}=0
$$

Here we use the subscript $n$ for the partial derivative by $t_{n}$.

Consider variables $s_{0}, s_{1}, s_{2}, \ldots$ and let $s_{0}:=s$. Let $F$ be a formal power series in the variables $t_{0}, t_{1}, \ldots, s_{0}, s_{1}, \ldots$ with the coefficients from $\mathbb{C}\left[u, u^{-1}\right]$. In [Bur14] we introduced the following equations:

$$
\frac{n+1}{u^{2}} F_{s_{n}}=\left(\frac{1}{2} \frac{\partial^{2}}{\partial t_{0}^{2}}+F_{0} \frac{\partial}{\partial t_{0}}+\frac{1}{2} F_{0}^{2}+\frac{1}{2} F_{0,0}+F_{0,0}^{c}\right) F_{s_{n-1}}, \quad n \geq 1 .
$$

We proved that the system (1.4)-(1.5) together with (1.7) has a unique solution for an arbitrary

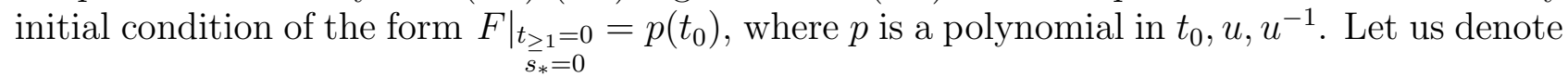
by $F^{o, e x t}$ a unique solution of this big system, that is specified by the initial condition

$$
\left.F\right|_{\substack{t_{\geq 1}=0 \\ s_{*}=0}}=0 .
$$


We call this solution the extended open potential. We obviously have

$$
\left.F^{o, e x t}\right|_{s_{\geq 1}=0}=F^{o} .
$$

The main observation of this paper is that the system of equations (1.4),(1.5) and (1.7) is in fact not new. If we rewrite it for the exponent $\exp (F)$, we get the system that coincides with the system of equations for the wave function of the KdV hierarchy. This allows us to derive an explicit formula for $F^{o, e x t}$ that we present in the next section.

1.3. Explicit formula for $F^{o, e x t}$. In this section we formulate the main result of the paper an explicit formula for the potential $F^{o, e x t}$.

Denote by $G_{z}$ the shift operator that acts on a power series $f\left(t_{0}, t_{1}, \ldots\right) \in \mathbb{C}\left[u, u^{-1}\right]\left[\left[t_{0}, t_{1}, \ldots\right]\right]$ as follows:

$$
G_{z}(f)\left(t_{0}, t_{1}, \ldots\right):=f\left(t_{0}-\frac{k_{0}}{z}, t_{1}-\frac{k_{1}}{u^{2} z^{3}}, t_{2}-\frac{k_{2}}{u^{4} z^{5}}, \ldots\right),
$$

where $k_{n}:=(2 n-1) ! !$ and, by definition, we put $(-1) ! !:=1$. Let

$$
\xi\left(t_{0}, t_{1}, \ldots, s_{0}, s_{1}, \ldots ; z\right):=\sum_{i \geq 0} \frac{t_{i} u^{2 i} z^{2 i+1}}{(2 i+1) ! !}+\sum_{i \geq 0} \frac{s_{i} u^{2 i+1} z^{2 i+2}}{2^{i+1}(i+1) !} .
$$

Let us also introduce a Laurent series $D(z) \in \mathbb{C}\left[u, u^{-1}\right]\left[\left[z^{-1}\right]\right]$. Define the numbers $a_{0}, a_{1}, \ldots$ and $d_{0}, d_{1}, d_{2}, \ldots$ by

$$
\begin{aligned}
& a_{n}:=(-1)^{n} \frac{(6 n) !}{288^{n}(2 n) !(3 n) !}, \\
& d_{n}:=\sum_{i=0}^{n} 3^{i}\left|a_{n-i}\right| \prod_{k=1}^{i}\left(n+\frac{1}{2}-k\right) .
\end{aligned}
$$

The Laurent series $D(z)$ is defined by $D(z):=1+\sum_{i \geq 1} \frac{d_{i}}{u^{2 i} z^{3 i}}$. Now we are ready to formulate our main theorem.

Theorem 1.1. We have

$$
\exp \left(F^{o, e x t}\right)=\operatorname{Coef}_{z^{0}}\left[D(z) \frac{G_{z}\left(\exp \left(F^{c}\right)\right)}{\exp \left(F^{c}\right)} \exp (\xi)\right] .
$$

We see that $D(z) \frac{G_{z}\left(\exp \left(F^{c}\right)\right)}{\exp \left(F^{c}\right)}$ is a power series in $z^{-1}$. On the other hand, $\exp (\xi)$ is a power series in $z$. In general one can have problems with the multiplication of two such series. In our case the issue can be solved as follows. Let us introduce a gradation in the ring $\mathbb{C}\left[u, u^{-1}\right]\left[\left[t_{0}, t_{1}, \ldots, s_{0}, s_{1}, \ldots\right]\right]$ assigning to $t_{i}$ degree $2 i+1$ and to $s_{i}$ degree $2 i+2$. Since the degree of the coefficient of $z^{i}$ in $\exp (\xi)$ grows as $i$ grows, the product in the square brackets on the right-hand side of $(\underline{1.10})$ is well-defined.

1.4. Extended open Virasoro equations. Here we recall the open Virasoro equations from [PST14] and construct Virasoro type equations for the extended potential $F^{o, e x t}$.

The Virasoro operators $L_{n}, n \geq-1$, are defined as follows:

$$
\begin{array}{r}
L_{n}:=\sum_{i \geq 0} \frac{(2 i+2 n+1) ! !}{2^{n+1}(2 i-1) ! !}\left(t_{i}-\delta_{i, 1}\right) \frac{\partial}{\partial t_{i+n}}+\frac{u^{2}}{2} \sum_{i=0}^{n-1} \frac{(2 i+1) ! !(2 n-2 i-1) ! !}{2^{n+1}} \frac{\partial^{2}}{\partial t_{i} \partial t_{n-1-i}} \\
+\delta_{n,-1} \frac{t_{0}^{2}}{2 u^{2}}+\delta_{n, 0} \frac{1}{16} .
\end{array}
$$

The Witten potential $F^{c}$ satisfies the so-called Virasoro equations (see [DVV91]):

$$
L_{n} \exp \left(F^{c}\right)=0, \quad n \geq-1 .
$$

The equation $L_{-1} \tau^{c}=0$ is called the string equation. 
In [PST14] the authors introduced the following modified operators:

$$
\mathcal{L}_{n}:=L_{n}+u^{n} s \frac{\partial^{n+1}}{\partial s^{n+1}}+\frac{3 n+3}{4} u^{n} \frac{\partial^{n}}{\partial s^{n}}, \quad n \geq-1,
$$

and conjectured that

$$
\mathcal{L}_{n} \exp \left(F^{o}+F^{c}\right)=0, \quad n \geq-1 .
$$

These equations are called the open Virasoro equations. We proved this conjecture in [Bur14]. Introduce operators $\mathcal{L}_{n}^{e x t}, n \geq-1$, by

$$
\mathcal{L}_{n}^{e x t}:=L_{n}+\sum_{i \geq 0} \frac{(i+n+1) !}{i !} s_{i} \frac{\partial}{\partial s_{n+i}}+\frac{3(n+1) !}{4} u \frac{\partial}{\partial s_{n-1}}+\delta_{n,-1} u^{-1} s+\delta_{n, 0} \frac{3}{4} .
$$

Here we, by definition, put $\frac{\partial}{\partial s_{-2}}:=\frac{\partial}{\partial s_{-1}}:=0$. Our second result is the following theorem.

Theorem 1.2. For any $n \geq-1$ we have

$$
\mathcal{L}_{n}^{e x t} \exp \left(F^{o, e x t}+F^{c}\right)=0 .
$$

We call these equations the extended open Virasoro equations. At first glance, our new Virasoro operators $\mathcal{L}_{n}^{\text {ext }}$ look quite different from the operators $\mathcal{L}_{n}$. Actually, we will show that the open Virasoro equations (1.12) can be easily derived from the extended open Virasoro equations (1.13).

1.5. Organization of the paper. In Section 2 we recall all necessary facts about the KdV hierarchy. In Section 3 we show that the exponent $\exp \left(F^{o, e x t}\right)$ satisfies the same equations as the wave function of the KdV hierarchy. Section 4 is devoted to the proof of Theorem 1.1. In Section 5 we prove Theorem 1.2. Appendix A contains some technical statements that we use in Sections 3 and 4 .

1.6. Acknowledgments. We thank G. Carlet, R. Pandharipande, S. Shadrin, J. Solomon and R. Tessler for discussions related to the work presented here.

We would like to thank the anonymous referee for valuable remarks and suggestions that allowed us to improve the exposition of this paper.

This work was supported by grant ERC-2012-AdG-320368-MCSK in the group of R. Pandharipande at ETH Zurich, by the Russian Federation Government grant no. 2010-220-01-077 (ag. no. 11.634.31.0005), the grants RFFI 13-01-00755 and NSh-4850.2012.1.

Part of the work was completed during the visit of the author to the Einstein Institute of Mathematics of the Hebrew University of Jerusalem in 2014.

1.7. Note added in proof. We would like to mention several papers Ale14a, Ale14b, BY14, BH15, BT15] that are related to the present work and that appeared while this paper was under consideration in the journal. In [Ale14b] (see also Ale14a]) A. Alexandrov used Theorem 1.1 and derived a matrix model for the exponent $e^{F^{o, e x t}+F^{c}}$. He also proved that after a certain simple change of variables the exponent $e^{F^{o, e x t}+F^{c}}$ becomes a tau-function of the KP hierarchy. In [BY14] M. Bertola and D. Yang suggested a natural candidate for the generating series of open $r$-spin intersection numbers and found a generalization of our Theorem 1.1. In [BT15], together with R. Tessler we proved the main conjecture from [PST14] that the generating series of the open intersection numbers satisfies the open KdV equations.

\section{LAX FORM OF THE KDV HIERARCHY}

Here we recall the Lax formalism in the theory of the KdV hierarchy. The book [Dic03] is a very good reference to this subject. Our notations are slightly different, because we rescale the flows of the KdV hierarchy and also we insert the formal variable $u$ in the coefficients of the equations of the hierarchy. Similar notations are used in [CvdLS14]. 
2.1. Pseudo-differential operators. A pseudo-differential operator $A$ is a Laurent series

$$
A=\sum_{n=-\infty}^{m} a_{n}(t) \partial_{x}^{n}
$$

where $m$ is an arbitrary integer and $a_{n}$ are formal power series in $t_{0}, t_{1}, \ldots$ with the coefficients from $\mathbb{C}\left[u, u^{-1}\right]$. We will always identify $t_{0}$ with $x$. Let

$$
A_{+}:=\sum_{n=0}^{m} a_{n} \partial_{x}^{n}, \quad \text { and } \quad A_{-}:=A-A_{+} .
$$

The product of pseudo-differential operators is defined by the following commutation rule:

$$
\partial_{x}^{k} \circ f:=\sum_{l=0}^{\infty} \frac{k(k-1) \ldots(k-l+1)}{l !} \frac{\partial^{l} f}{\partial x^{l}} \partial_{x}^{k-l},
$$

where $k \in \mathbb{Z}$ and $f \in \mathbb{C}\left[u, u^{-1}\right]\left[\left[t_{0}, t_{1}, \ldots\right]\right]$. The conjugate $A^{*}$ of a pseudo-differential operator $A=\sum_{n=-\infty}^{m} a_{n} \partial_{x}^{n}$ is defined by

$$
A^{*}:=\sum_{n=-\infty}^{m}(-1)^{n} \partial_{x}^{n} \circ a_{n} .
$$

It is not hard to check that, for any pseudo-differential operator $P$ of the form $P=1+$ $\sum_{n \geq 1} p_{n}(t) \partial_{x}^{-n}$, there exists a unique inverse operator $P^{-1}$ of the same form $P^{-1}=1+$ $\sum_{n \geq 1} \widetilde{p}_{n}(t) \partial_{x}^{-n}$.

Let $m \geq 2$. Consider a pseudo-differential operator $A$ of the form

$$
A=\partial_{x}^{m}+\sum_{n=1}^{\infty} a_{n} \partial_{x}^{m-n} .
$$

It is not hard to see that there exists a unique pseudo-differential operator $A^{\frac{1}{m}}$ of the form

$$
A^{\frac{1}{m}}=\partial_{x}+\sum_{n=1}^{\infty} \widetilde{a}_{n} \partial_{x}^{m-n}
$$

such that $\left(A^{\frac{1}{m}}\right)^{m}=A$. This operator is called the $m$-th root of $A$.

\subsection{Lax form of the $\mathrm{KdV}$ hierarchy. Let}

$$
L:=\partial_{x}^{2}+2 w, \quad w \in \mathbb{C}\left[u, u^{-1}\right]\left[\left[t_{0}, t_{1}, \ldots\right]\right] .
$$

It is easy to check that, for any $n \geq 1$, the commutator $\left[\left(L^{n+\frac{1}{2}}\right)_{+}, L\right]$ doesn't have terms with positive powers of $\partial_{x}$. The $\mathrm{KdV}$ hierarchy is the following system of partial differential equations for the power series $w$ :

$$
\frac{\partial L}{\partial t_{n}}=\frac{u^{2 n}}{(2 n+1) ! !}\left[\left(L^{n+\frac{1}{2}}\right)_{+}, L\right], \quad n=1,2, \ldots
$$

This form of the KdV equations is called the Lax form.

2.3. Dressing operator. Let $L$ be a solution of the Lax equations (2.1). Then there exists a pseudo-differential operator $P$ of the form

$$
P=1+\sum_{n \geq 1} p_{n}(t) \partial_{x}^{-n}
$$


such that

$$
\begin{aligned}
& L=P \circ \partial_{x}^{2} \circ P^{-1}, \quad \text { and } \\
& \frac{\partial P}{\partial t_{n}}=-\frac{u^{2 n}}{(2 n+1) ! !}\left(L^{n+\frac{1}{2}}\right)_{-} \circ P, \quad n=1,2 \ldots
\end{aligned}
$$

The operator $P$ is called the dressing operator and the Laurent series

$$
\widehat{P}(t ; z):=1+\sum_{n \geq 1} p_{n}(t) z^{-n}
$$

is called the symbol of the dressing operator $P$. Equations (2.4) are called the Sato-Wilson equations.

We also have the following converse statement. Let $P$ be a pseudo-differential operator of the form (2.2). Suppose that the operator $P$ satisfies the equations

$$
\frac{\partial P}{\partial t_{n}}=-\frac{u^{2 n}}{(2 n+1) ! !}\left(P \circ \partial_{x}^{2 n+1} \circ P^{-1}\right)_{-} \circ P, \quad n=1,2 \ldots
$$

and has the property $\left(P \circ \partial_{x}^{2} \circ P^{-1}\right)_{-}=0$. Define an operator $L$ by (2.3). Then the operator $L$ has the form $L=\partial_{x}^{2}+2 w$ for some power series $w \in \mathbb{C}\left[u, u^{-1}\right]\left[\left[t_{0}, t_{1}, \ldots\right]\right]$ and it satisfies the Lax equations (2.1).

2.4. Wave function. Let $P=1+\sum_{n \geq 1} p_{n}(t) \partial_{x}^{-n}$ be the dressing operator for some solution $L$ of the Lax equations (2.1). It is convenient to introduce variables $t_{n}$ with $n \in \frac{1}{2}+\mathbb{Z}_{\geq 0}$ and put $t_{\frac{1}{2}+n}:=s_{n}$. Then the power series $\xi\left(t_{*}, s_{*} ; z\right)$, that we defined in Section 1.3, can be written as follows:

$$
\xi(t ; z)=\sum_{i \in \frac{1}{2} \mathbb{Z} \geq 0} \frac{t_{i} u^{2 i} z^{2 i+1}}{(2 i+1) ! !}
$$

Here $(2 n) ! !:=2^{n} n !$. The product

$$
\psi(t ; z):=\widehat{P}(t ; z) \exp (\xi(t ; z))
$$

is called the wave function of the $\mathrm{KdV}$ hierarchy. It satisfies the following equations:

$$
\begin{aligned}
\frac{\partial \psi}{\partial t_{n}} & =\frac{u^{2 n}}{(2 n+1) ! !}\left(L^{n+\frac{1}{2}}\right)_{+} \psi, \quad n \in \frac{1}{2} \mathbb{Z}_{\geq 1} ; \\
L \psi & =z^{2} \psi
\end{aligned}
$$

2.5. Tau-function. Let $L$ be a solution of the Lax equations (2.1). Then there exists a power series $\tau \in \mathbb{C}\left[u, u^{-1}\right]\left[\left[t_{0}, t_{1}, \ldots\right]\right]$ such that $\left.\tau\right|_{t_{*}=0}=1$ and the power series

$$
\frac{G_{z}(\tau)}{\tau} \in \mathbb{C}\left[u, u^{-1}\right]\left[\left[z^{-1}, t_{0}, t_{1}, \ldots\right]\right]
$$

is the symbol of the dressing operator for the operator $L$. The power series $\tau$ is called the tau-function of the KdV hierarchy. The tau-function has the following property:

$$
\frac{\partial^{2} \ln \tau}{\partial t_{0}^{2}}=w
$$

2.6. Witten's conjecture. Let $L=\partial_{x}^{2}+2 F_{0,0}^{c}$. Witten's conjecture says that the operator $L$ is a solution of the Lax equations (2.1) and the exponent $\exp \left(F^{c}\right)$ is the corresponding taufunction. 


\section{Open KdV equations and the equations for the Wave Function of the KdV HIERARCHY}

In this section we show that the $\operatorname{exponent} \exp \left(F^{o, e x t}\right)$ satisfies equations (2.5) for the wave function of the KdV hierarchy.

Let $w:=F_{0,0}^{c}$ and $L:=\partial_{x}^{2}+2 w$. By Witten's conjecture, the operator $L$ is a solution of the Lax equations (2.1) and the exponent $\exp \left(F^{c}\right)$ is the corresponding tau-function. Recall that we have introduced the variables $t_{n}$ with $n \in \frac{1}{2}+\mathbb{Z}_{\geq 0}$ and put $t_{k+\frac{1}{2}}:=s_{k}$.

Theorem 3.1. For any $n \in \frac{1}{2} \mathbb{Z}_{\geq 0}$ we have

$$
\frac{\partial}{\partial t_{n}} \exp \left(F^{o, e x t}\right)=\frac{u^{2 n}}{(2 n+1) ! !}\left(L^{n+\frac{1}{2}}\right)_{+} \exp \left(F^{o, e x t}\right) .
$$

Recall that we identify $x$ with $t_{0}$.

Proof. The proof is by induction on $n$. Suppose $n$ is a half-integer, $n=k+\frac{1}{2}$. If $k=0$, then, by (1.4),

$$
\frac{\partial}{\partial t_{\frac{1}{2}}} F^{o, e x t}=\frac{\partial}{\partial s} F^{o, e x t}=u\left(\frac{1}{2} F_{0,0}^{o, e x t}+\frac{1}{2}\left(F_{0}^{o, e x t}\right)^{2}+w\right) .
$$

Therefore,

$$
\frac{\partial}{\partial t_{\frac{1}{2}}} \exp \left(F^{o, e x t}\right)=\frac{u}{2}\left(\partial_{x}^{2}+2 w\right) \exp \left(F^{o, e x t}\right) .
$$

This is exactly equation (3.1) for $n=\frac{1}{2}$.

Suppose $k$ is a positive integer. We compute that

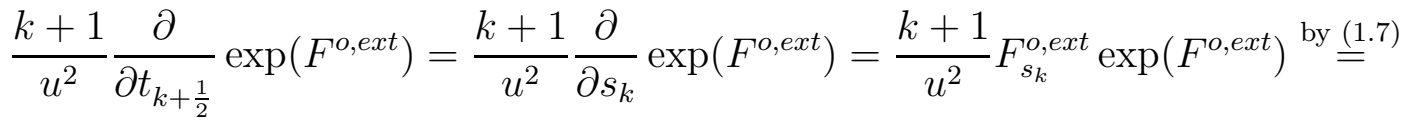

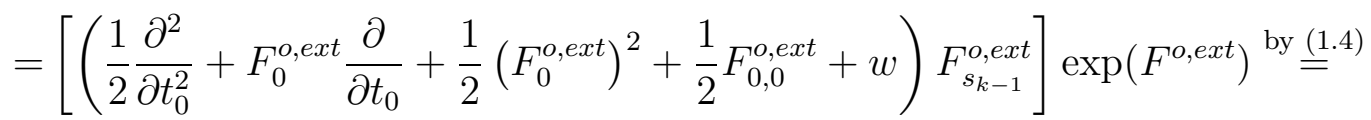

$$
\begin{aligned}
& =u^{-1}\left(F_{s, s_{k-1}}^{o, e x t}+F_{s}^{o, e x t} F_{s_{k-1}}^{o, e x t}\right) \exp \left(F^{o, e x t}\right)=u^{-1} \frac{\partial^{2}}{\partial s \partial s_{k-1}} \exp \left(F^{o, e x t}\right) \stackrel{\substack{\text { by the induction } \\
\text { assumption }}}{=} \\
& =u^{-1} \frac{\partial}{\partial s}\left[\frac{u^{2 k-1}}{(2 k) ! !} L^{k} \exp \left(F^{o, e x t}\right)\right] \text { by } \stackrel{(3.2)}{=} \frac{u^{2 k-1}}{2(2 k) ! !} L^{k+1} \exp \left(F^{o, e x t}\right) \text {. }
\end{aligned}
$$

Thus, equation (3.1) is proved for half-integers.

Suppose $n$ is an integer. For $n=0$ equation (3.1) is obvious. Suppose that $n \geq 1$. We have

$$
\begin{aligned}
\frac{2 n+1}{2} \frac{\partial}{\partial t_{n}} \exp \left(F^{o, e x t}\right)=\frac{2 n+1}{2} F_{n}^{o, e x t} \exp \left(F^{o, e x t}\right) \stackrel{\text { by } \underline{\underline{(1.2)}}}{=} \\
\quad=\left[u F_{s, n-1}^{o, e x t}+u F_{s}^{o, e x t} F_{n-1}^{o, e x t}+\frac{u^{2}}{2} F_{0, n-1}^{c} F_{0}^{o, e x t}-\frac{u^{2}}{4} F_{0,0, n-1}^{c}\right] \exp \left(F^{o, e x t}\right)= \\
\left.\quad=\left(u \frac{\partial^{2}}{\partial s \partial t_{n-1}}+\frac{u^{2}}{2} F_{0, n-1}^{c} \frac{\partial}{\partial t_{0}}-\frac{u^{2}}{4} F_{0,0, n-1}^{c}\right) \exp \left(F^{o, e x t}\right) \stackrel{\text { assumption and } \stackrel{u^{2}}{=} \text { (3.2) }}{2(2 n-1) ! !}\left(L^{n-\frac{1}{2}}\right)_{+} \circ L+\frac{u^{2}}{2} F_{0, n-1}^{c} \partial_{x}-\frac{u^{2}}{4} F_{0,0, n-1}^{c}\right) \exp \left(F^{o, e x t}\right) \stackrel{\text { by Lemma A.3. }}{=} \\
\quad=\frac{u^{2 n}}{2(2 n-1) ! !}\left(L^{n+\frac{1}{2}}\right)_{+} \exp \left(F^{o, e x t}\right) .
\end{aligned}
$$

The theorem is proved. 


\section{Proof of Theorem 1.1}

In this section we prove Theorem 1.1 and show that $\mathcal{L}_{-1}^{e x t} \exp \left(F^{o, e x t}+F^{c}\right)=0$.

Let $\tau^{c}:=\exp \left(F^{c}\right)$. By Witten's conjecture, $\tau^{c}$ is a tau-function of the KdV hierarchy. Therefore the series $\psi$, defined by

$$
\psi:=\frac{G_{z}\left(\tau^{c}\right)}{\tau^{c}} \exp (\xi),
$$

is the wave function of the $\mathrm{KdV}$ hierarchy. Let $w:=F_{0,0}^{c}, L:=\partial_{x}^{2}+2 w$ and

$$
H(t ; z):=G_{z}\left(\tau^{c}\right) \exp (\xi)=\tau^{c} \psi .
$$

The proof of Theorem 1.1 is based on the following proposition.

Proposition 4.1. We have $\mathcal{L}_{-1}^{\text {ext }} \operatorname{Coef}_{z^{0}}(D \cdot H)=0$.

Proof. It is easy to compute that

$$
\begin{aligned}
\mathcal{L}_{-1}^{e x t} H= & G_{z}\left(L_{-1} \tau^{c}\right) \exp (\xi)+ \\
& +\left(\sum_{i \geq 0} \frac{(2 i+1) ! !}{u^{2 i+2} z^{2 i+3}} G_{z}\left(F_{i}^{c}\right)+\sum_{i \geq 0} t_{i} \frac{u^{2 i-2} z^{2 i-1}}{(2 i-1) ! !}+\sum_{i \geq 0} s_{i} \frac{u^{2 i-1} z^{2 i}}{2^{i} i !}-\frac{1}{2 u^{2} z^{2}}-z\right) H, \\
\frac{1}{u^{2} z} \frac{\partial}{\partial z} H= & \left(\sum_{i \geq 0} \frac{(2 i+1) ! !}{u^{2 i+2} z^{2 i+3}} G_{z}\left(F_{i}^{c}\right)+\sum_{i \geq 0} t_{i} \frac{u^{2 i-2} z^{2 i-1}}{(2 i-1) ! !}+\sum_{i \geq 0} s_{i} \frac{u^{2 i-1} z^{2 i}}{2^{i} i !}\right) H .
\end{aligned}
$$

Since $L_{-1} \tau^{c}=0$, we get

$$
\frac{\mathcal{L}_{-1}^{e x t} H}{\tau^{c}}=\left(\tau^{c}\right)^{-1}\left(\frac{1}{u^{2} z} \frac{\partial}{\partial z}-\frac{1}{2 u^{2} z^{2}}-z\right) H=\left(\frac{1}{u^{2} z} \frac{\partial}{\partial z}-\frac{1}{2 u^{2} z^{2}}-z\right) \psi .
$$

Denote the operator $\frac{1}{u^{2} z} \frac{\partial}{\partial z}-\frac{1}{2 u^{2} z^{2}}-z$ by $S_{z}$. We see that we have to prove that

$$
\operatorname{Coef}_{z^{0}}\left(D \cdot S_{z} \psi\right)=0 \text {. }
$$

Let $\mathcal{F} \in \mathbb{C}\left[u, u^{-1}\right]\left[\left[t_{0}, t_{\frac{1}{2}}, t_{1}, t_{\frac{3}{2}}, \ldots\right]\right]$. Consider the following system:

$$
\frac{\partial \mathcal{F}}{\partial t_{n}}=\frac{u^{2 n}}{(2 n+1) ! !}\left(L^{n+\frac{1}{2}}\right)_{+} \mathcal{F}, \quad n \in \frac{1}{2} \mathbb{Z}_{\geq 1}
$$

From (2.5) it follows that the series $\operatorname{Coef}_{z^{0}}\left(D \cdot S_{z} \psi\right)$ is a solution of this system. Therefore, in order to prove (4.2), it is sufficient to prove that

$$
\left.\operatorname{Coef}_{z^{0}}\left(D \cdot S_{z} \psi\right)\right|_{t_{>0}=0}=0 \text {. }
$$

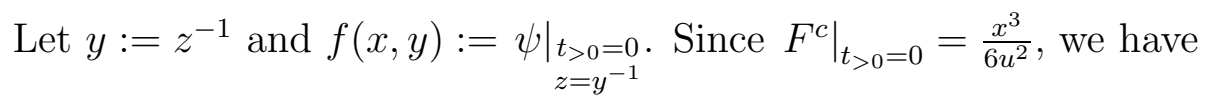

$$
f(x, y)=\frac{\exp \left(F^{c}\left(x-y,-\frac{y^{3}}{u^{2}},-\frac{3 ! ! y^{5}}{u^{4}}, \ldots\right)\right)}{\exp \left(\frac{x^{3}}{6 u^{2}}\right)} \exp \left(\frac{x}{y}\right) .
$$

Denote by $S_{y}$ the operator $-S_{z}$, rewritten in the $y$-coordinate. We have

$$
S_{y}=\frac{y^{3}}{u^{2}} \partial_{y}+\frac{y^{2}}{2 u^{2}}+\frac{1}{y}
$$

We have to prove that

$$
\operatorname{Coef}_{y^{0}}\left(D \cdot S_{y} f\right)=0 .
$$

In the next three lemmas we describe several properties of the series $f$.

Lemma 4.2. The series $f$ satisfies the homogeneity condition $\left(x \frac{\partial}{\partial x}+y \frac{\partial}{\partial y}+\frac{3}{2} u \frac{\partial}{\partial u}\right) f=0$. 
Proof. Let $O:=x \frac{\partial}{\partial x}+y \frac{\partial}{\partial y}+\frac{3}{2} u \frac{\partial}{\partial u}$. A correlator $\left\langle\tau_{d_{1}} \ldots \tau_{d_{n}}\right\rangle_{g}^{c}$ can be non-zero, only if $d_{1}+\ldots+$ $d_{n}=3 g-3+n$. Therefore we have

$$
\left(\sum_{i \geq 0}(1-i) t_{i} \frac{\partial}{\partial t_{i}}+\frac{3}{2} u \frac{\partial}{\partial u}\right) F^{c}=0 .
$$

This equation easily implies that $O F^{c}\left(x-y,-\frac{y^{3}}{u^{2}},-\frac{3 ! ! y^{5}}{u^{4}}, \ldots\right)=0$. Since $O\left(\frac{x^{3}}{6 u^{2}}\right)=O\left(\frac{x}{y}\right)=0$, we get $O f=0$.

Lemma 4.3. We have $\frac{\partial f}{\partial x}=S_{y} f$.

Proof. By (4.1), $\left(\tau^{c}\right)^{-1} \mathcal{L}_{-1}^{e x t} H=S_{z} \psi$. It is easy to see that

$$
\begin{aligned}
\left.\left(\left(\tau^{c}\right)^{-1} \mathcal{L}_{-1}^{e x t} H\right)\right|_{t_{>0}=0}=e^{-\frac{t_{0}^{3}}{6 u^{2}}}\left(-\frac{\partial}{\partial t_{0}}+\frac{t_{0}^{2}}{2 u^{2}}\right) & \left(\left.H\right|_{t_{>0}=0}\right)= \\
& =-\frac{\partial}{\partial t_{0}}\left(\left.e^{-\frac{t_{0}^{3}}{6 u^{2}}} H\right|_{t_{>0}=0}\right)=-\left.\left(\frac{\partial \psi}{\partial t_{0}}\right)\right|_{t_{>0}=0} .
\end{aligned}
$$

Therefore, $\frac{\partial f}{\partial x}=S_{y} f$.

Now we want to compute $\left.f\right|_{x=0}$. Recall that the numbers $a_{n}, n \geq 0$, are defined by

$$
a_{n}:=(-1)^{n} \frac{(6 n) !}{288^{n}(2 n) !(3 n) !} .
$$

Introduce the power series $A(y)$ by

$$
A(y):=1+\sum_{i \geq 1} a_{i} \frac{y^{3 i}}{u^{2 i}} .
$$

Lemma 4.4. We have $\left.f\right|_{x=0}=A(y)$.

Proof. It is possible to get the proof from the results of [Kon92] or [KS91], but we decide to present a simple direct argument here. First of all, from Lemma 4.2 it follows that the series $\left.f\right|_{x=0}$ has the form

$$
\left.f\right|_{x=0}=1+\sum_{i \geq 1} f_{i} \frac{y^{3 i}}{u^{2 i}}
$$

where $f_{i}$ are some complex coefficients. From Lemma 4.3 it follows that $\frac{\partial^{2}}{\partial x^{2}} f=S_{y}^{2} f$. By (2.6)

$$
\left(\partial_{x}^{2}+2 x\right) f=\frac{1}{y^{2}} f
$$

So we get

$$
S_{y}^{2}\left(\left.f\right|_{x=0}\right)=\frac{1}{y^{2}}\left(\left.f\right|_{x=0}\right) .
$$

From Lemma A.4 it follows that $\left.f\right|_{x=0}=A(y)$.

In order to prove equation (4.4), we have to prove that, for any $n \geq 0$, we have

$$
\left.\operatorname{Coef}_{y^{0}}\left(D \cdot \frac{\partial^{n}}{\partial x^{n}} S_{y} f\right)\right|_{x=0}=0 .
$$

By Lemmas 4.3 and 4.4, we have

$$
\left.\operatorname{Coef}_{y^{0}}\left(D \cdot \frac{\partial^{n}}{\partial x^{n}} S_{y} f\right)\right|_{x=0}=\operatorname{Coef}_{y^{0}}\left(D \cdot S_{y}^{n+1} A(y)\right) .
$$

Let $\widehat{S}_{y}:=-\frac{y^{3}}{u^{2}} \partial_{y}-\frac{3 y^{2}}{2 u^{2}}+\frac{1}{y}$. 
Lemma 4.5. For any two Laurent series $\left.p, q \in \mathbb{C}\left[u, u^{-1}\right]\left[y^{-1}, y\right]\right]$ we have

$$
\operatorname{Coef}_{y^{0}}\left(p \cdot S_{y} q\right)=\operatorname{Coef}_{y^{0}}\left(\widehat{S}_{y} p \cdot q\right) .
$$

Proof. We have

$$
\begin{aligned}
& \operatorname{Coef}_{y^{0}}\left(p \cdot \frac{y^{3}}{u^{2}} \frac{\partial q}{\partial y}\right)=\operatorname{Coef}_{y^{-1}}\left(p \cdot \frac{y^{2}}{u^{2}} \frac{\partial q}{\partial y}\right)= \\
& =\operatorname{Coef}_{y^{-1}}\left(\frac{\partial}{\partial y}\left(p \cdot \frac{y^{2}}{u^{2}} q\right)\right)-\operatorname{Coef}_{y^{-1}}\left(\frac{\partial}{\partial y}\left(\frac{y^{2}}{u^{2}} p\right) \cdot q\right)=\operatorname{Coef}_{y^{0}}\left(\left(-\frac{y^{3}}{u^{2}} \frac{\partial}{\partial y}-\frac{2 y^{2}}{u^{2}}\right) p \cdot q\right) .
\end{aligned}
$$

The lemma is clear now.

Using this lemma we get

$$
\operatorname{Coef}_{y^{0}}\left(D \cdot S_{y}^{n+1} A(y)\right)=\operatorname{Coef}_{y^{0}}\left(\widehat{S}_{y} D \cdot S_{y}^{n} A(y)\right) \stackrel{\text { by Lemma }}{=} \operatorname{Coef}_{y^{0}}\left(\frac{A(-y)}{y} \cdot S_{y}^{n} A(y)\right) .
$$

Define the power series $B(y)$ by $B(y):=y S_{y} A(y)$. It is easy to see that

$$
B(y)=1+\sum_{i \geq 1} b_{i} \frac{y^{3 i}}{u^{2 i}}, \quad \text { where } \quad b_{i}=-\frac{6 i+1}{6 i-1} a_{i} .
$$

By Lemma A.7, we have

$$
\operatorname{Coef}_{y^{0}}\left(\frac{A(-y)}{y} \cdot S_{y}^{n} A(y)\right)=\operatorname{Coef}_{y^{0}}\left(\frac{Q_{n}}{y} A(-y) A(y)+\frac{R_{n}}{y^{2}} A(-y) B(y)\right),
$$

where $Q_{n}, R_{n} \in \mathbb{C}\left[u^{-1}, y^{-1}\right]$ are even Laurent polynomials in $y$. Since $A(-y) A(y)$ is even, we get $\operatorname{Coef}_{y^{0}}\left(\frac{Q_{n}}{y} A(-y) A(y)\right)=0$. We also have the following identity (see e.g. [PPZ13, page $36])$ :

$$
A(y) B(-y)+A(-y) B(y)=2 .
$$

Therefore, $\operatorname{Coef}_{y^{0}}\left(\frac{R_{n}}{y^{2}} A(-y) B(y)\right)=0$. We conclude that the right-hand side of (4.5) is equal to zero. The proposition is proved.

Let us prove Theorem 1.1. By equation (2.5) and Theorem 3.1, the both series $\operatorname{Coef}_{z^{0}}(D \cdot \psi)$ and $\exp \left(F^{o, e x t}\right)$ are solutions of system (4.3). Therefore it is sufficient to check that

$$
\left.\operatorname{Coef}_{z^{0}}(D \cdot \psi)\right|_{t_{>0}=0}=\left.\exp \left(F^{o, e x t}\right)\right|_{t_{>0}} .
$$

Since the potential $F^{o, e x t}$ satisfies the initial condition (1.8), it remains to prove that

$$
\left.\operatorname{Coef}_{z^{0}}(D \cdot \psi)\right|_{t_{>0}=0}=1 \text {. }
$$

We can easily see that $\left.\operatorname{Coef}_{z^{0}}(D \cdot \psi)\right|_{t_{*}=0}=1$. Proposition 4.1 and the string equation $L_{-1} \tau^{c}=$ 0 imply that

$$
\left(-\frac{\partial}{\partial t_{0}}+\sum_{n \in \frac{1}{2} \mathbb{Z}_{\geq 0}} t_{n+1} \frac{\partial}{\partial t_{n}}+u^{-1} t_{\frac{1}{2}}\right) \operatorname{Coef}_{z^{0}}(D \cdot \psi)=0 .
$$

Thus, $\frac{\partial}{\partial t_{0}}\left(\left.\operatorname{Coef}_{z^{0}}(D \cdot \psi)\right|_{t_{>0}=0}\right)=0$, and the theorem is proved.

Obviously, Proposition 4.1 and Theorem 1.1 imply the following corollary.

Corollary 4.6. We have $\mathcal{L}_{-1}^{e x t} \exp \left(F^{o, e x t}+F^{c}\right)=0$.

\section{Extended open Virasoro equations}

In this section we prove Theorem 1.2 and show that the open Virasoro equations (1.12) can be easily derived from it. 
5.1. Proof of Theorem 1.2. By Corollary 4.6, we already know that $\mathcal{L}_{-1}^{e x t} \exp \left(F^{o, e x t}+F^{c}\right)=0$. By Theorem 1.1, we have $\exp \left(F^{o, e x t}+F^{c}\right)=\operatorname{Coef}_{z^{0}}(D \cdot H)$. A direct computation shows that, for any $n \geq-1$, we have

$$
\mathcal{L}_{n}^{e x t} H=G_{z}\left(L_{n} \tau^{c}\right) \exp (\xi)+\frac{u^{2 n+2} z^{2 n+2}}{2^{n+1}}\left(\mathcal{L}_{-1}^{e x t} H-G_{z}\left(L_{-1} \tau^{c}\right) \exp (\xi)\right)+\frac{n+1}{2^{n}} u^{2 n} z^{2 n} H .
$$

By equation (2.6),$L \psi=z^{2} \psi$. Using also the closed Virasoro equations (1.11), we get

$$
\mathcal{L}_{n}^{e x t} H=\frac{u^{2 n+2}}{2^{n+1}}\left(\mathcal{L}_{-1}^{e x t} \circ \tau^{c} \circ L^{n+1} \circ\left(\tau^{c}\right)^{-1}\right) H+\frac{u^{2 n}(n+1)}{2^{n}}\left(\tau^{c} \circ L^{n} \circ\left(\tau^{c}\right)^{-1}\right) H .
$$

Let $\widetilde{\mathcal{L}}_{-1}^{\text {ext }}:=\mathcal{L}_{-1}^{\text {ext }}-\frac{x^{2}}{2 u^{2}}$. From the string equation $L_{-1} \tau^{c}=0$ it follows that

$$
\mathcal{L}_{-1}^{e x t} \circ \tau^{c}=\tau^{c} \circ \widetilde{\mathcal{L}}_{-1}^{\text {ext }}, \quad\left[\widetilde{\mathcal{L}}_{-1}^{e x t}, L\right]=-\frac{2}{u^{2}} .
$$

Combining these equations with equation (5.1), we get

$$
\begin{aligned}
\mathcal{L}_{n}^{e x t} H & =\frac{u^{2 n+2}}{2^{n+1}}\left(\tau^{c} \circ \widetilde{\mathcal{L}}_{-1}^{e x t} \circ L^{n+1} \circ\left(\tau^{c}\right)^{-1}\right) H+\frac{u^{2 n}(n+1)}{2^{n}}\left(\tau^{c} \circ L^{n} \circ\left(\tau^{c}\right)^{-1}\right) H= \\
& =\frac{u^{2 n+2}}{2^{n+1}}\left(\tau^{c} \circ L^{n+1} \circ \widetilde{\mathcal{L}}_{-1}^{e x t} \circ\left(\tau^{c}\right)^{-1}\right) H=\frac{u^{2 n+2}}{2^{n+1}}\left(\tau^{c} \circ L^{n+1} \circ\left(\tau^{c}\right)^{-1}\right) \mathcal{L}_{-1}^{e x t} H .
\end{aligned}
$$

Since $\exp \left(F^{o, e x t}+F^{c}\right)=\operatorname{Coef}_{z^{0}}(D \cdot H)$, we obtain

$$
\mathcal{L}_{n}^{e x t} \exp \left(F^{o, e x t}+F^{c}\right)=\frac{u^{2 n+2}}{2^{n+1}}\left(\tau^{c} \circ L^{n+1} \circ\left(\tau^{c}\right)^{-1}\right) \mathcal{L}_{-1}^{e x t} \exp \left(F^{o, e x t}+F^{c}\right)=0 .
$$

The extended open Virasoro equations are proved.

5.2. Open Virasoro equations from the extended open Virasoro equations. Let us derive the open Virasoro equations (1.12) from the extended open Virasoro equations (1.13). We have $F^{o}=\left.F^{o, e x t}\right|_{s_{\geq 1}=0}$. From Theorem 3.1 it follows that

$$
\frac{\partial}{\partial s_{n}} \exp \left(F^{o, e x t}\right)=\frac{u^{n}}{(n+1) !} \frac{\partial^{n+1}}{\partial s^{n+1}} \exp \left(F^{o, e x t}\right) .
$$

We see that

$$
\left.\left(\mathcal{L}_{n}^{e x t} \exp \left(F^{o, e x t}+F^{c}\right)\right)\right|_{s_{\geq 1}=0}=\left.\left(\mathcal{L}_{n} \exp \left(F^{o, e x t}+F^{c}\right)\right)\right|_{s_{\geq 1}=0}=\mathcal{L}_{n} \exp \left(F^{o}+F^{c}\right) .
$$

Theorem 1.2 implies that $\mathcal{L}_{n} \exp \left(F^{o}+F^{c}\right)=0$. The open Virasoro equations are proved.

\section{Appendix A. Technical lemmas}

In this section we collect several technical facts and lemmas that we used in Sections 3 and 4 , In Section A.1 we list some properties of a tau-function of the KdV hierarchy. Section A.2 is devoted to properties of the power series $A, B$ and $D$.

A.1. Tau-function. Let $L=\partial_{x}^{2}+2 w$ be a solution of the Lax equations (2.1) and $\tau$ be the tau-function. Denote the $\log a r i t h m \ln \tau$ by $F$.

A.1.1. Second derivatives $F_{i, j}$. Suppose $f$ is a power series in $t_{0}, t_{1}, \ldots$ with the coefficients from $\mathbb{C}\left[u, u^{-1}\right]$. We will say that the power series $f$ is a differential polynomial in $w$, if it can be expressed as a polynomial in $w, w_{x}, w_{x x}, \ldots$ with the coefficients from $\mathbb{C}\left[u, u^{-1}\right]$. We will sometimes denote $\partial_{x}^{i} w$ by $w_{i}$.

Lemma A.1 (See e.g. Dic03]). For any $i, j \geq 0$ there exists a universal polynomial $P_{i, j} \in$ $\mathbb{C}\left[u, u^{-1}\right]\left[v_{0}, v_{1}, v_{2}, \ldots\right]$ in formal variables $v_{0}, v_{1}, v_{2}, \ldots$ such that

$$
F_{i, j}=\left.P_{i, j}\right|_{v_{k}=w_{k}} .
$$


The polynomial $P_{i, j}$ is universal in the sense, that it doesn't depend on a solution $L$ of the Lax equations and on the choice of a $\tau$-function. The polynomials $P_{i, j}$ have the property

$$
\left.P_{i, j}\right|_{v_{*}=0}=0
$$

For example, $P_{0,0}=v_{0}$. Therefore, $F_{0,0}=w$.

A.1.2. Recursion for the operators $\left(L^{n+\frac{1}{2}}\right)_{+}$.

Lemma A.2. For any $n \in \mathbb{Z}_{\geq 0}$ we have

$$
L^{n+\frac{1}{2}}=\left(L^{n+\frac{1}{2}}\right)_{+}+\frac{(2 n+1) ! !}{u^{2 n}} F_{0, n} \partial_{x}^{-1}-\frac{1}{2} \frac{(2 n+1) ! !}{u^{2 n}} F_{0,0, n} \partial_{x}^{-2}+\ldots
$$

Proof. Let

$$
L^{n+\frac{1}{2}}=\left(L^{n+\frac{1}{2}}\right)_{+}+a_{n} \partial_{x}^{-1}+b_{n} \partial_{x}^{-2}+\ldots
$$

We have $\left[L^{n+\frac{1}{2}}, L\right]=0$, therefore,

$$
\left[\left(L^{n+\frac{1}{2}}\right)_{+}+a_{n} \partial_{x}^{-1}+b_{n} \partial_{x}^{-2}, \partial_{x}^{2}+2 w\right]_{+}=0
$$

Since $w=F_{0,0}$, we have $\frac{u^{2 n}}{(2 n+1) ! !}\left[\left(L^{n+\frac{1}{2}}\right)_{+}, L\right]=2 F_{0,0, n}$. Expanding (A.2) we get

$$
2 \frac{(2 n+1) ! !}{u^{2 n}} F_{0,0, n}-2 \partial_{x} a_{n}=0 .
$$

Note that $a_{n}$ is a universal differential polynomial in $w, w_{x}, w_{x x}, \ldots$ It is also easy to see that $a_{n}$ is equal to zero, if $w$ is zero. Therefore, we conclude that $a_{n}=\frac{(2 n+1) ! !}{u^{2 n}} F_{0, n}$.

Let us compute $b_{n}$. We have

$$
\left(L^{n+\frac{3}{2}}\right)_{+}=\left(L^{n+\frac{1}{2}}\right)_{+} \circ L+a_{n} \partial_{x}+b_{n} .
$$

Note that for any non-negative integer $k$ we have

$$
\left(L^{\frac{k}{2}}\right)_{+}^{*}=(-1)^{k}\left(L^{\frac{k}{2}}\right)_{+} .
$$

Thus,

$$
-\left(L^{n+\frac{3}{2}}\right)_{+}=-L \circ\left(L^{n+\frac{1}{2}}\right)_{+}-\partial_{x} a_{n}-a_{n} \partial_{x}+b_{n} .
$$

Summing (A.3) and (A.4), we obtain

$$
\left[\left(L^{n+\frac{1}{2}}\right)_{+}, L\right]-\partial_{x} a_{n}+2 b_{n}=0
$$

Thus, $b_{n}=-\frac{1}{2} \frac{(2 n+1) ! !}{u^{2 n}} F_{0,0, n}$. The lemma is proved.

Lemma A.3. For any $n \in \mathbb{Z}_{\geq 1}$ we have

$$
\left(L^{n+\frac{1}{2}}\right)_{+}=\left(L^{n-\frac{1}{2}}\right)_{+} \circ L+\frac{(2 n-1) ! !}{u^{2 n-2}} F_{0, n-1} \partial_{x}-\frac{1}{2} \frac{(2 n-1) ! !}{u^{2 n-2}} F_{0,0, n-1} .
$$

Proof. This is just equation (A.3) with $n$ replaced by $n-1$. 
A.2. Properties of the series $A, B$ and $D$. Recall that $S_{y}:=\frac{y^{3}}{u^{2}} \frac{\partial}{\partial y}+\frac{y^{2}}{2 u^{2}}+\frac{1}{y}, \widehat{S}_{y}:=-\frac{y^{3}}{u^{2}} \frac{\partial}{\partial y}-$ $\frac{3 y^{2}}{2 u^{2}}+\frac{1}{y}, A(y):=1+\sum_{i \geq 1} a_{i} \frac{y^{3 i}}{u^{2 i}}$ and $D:=1+\sum_{i \geq 1} d_{i} \frac{y^{3 i}}{u^{2 i}}$.

Let $C$ be a power series of the form $C=1+\sum_{i \geq 1} c_{i} \frac{y^{3 i}}{u^{2 i}}$, where $c_{i}$ are some complex coefficients. Consider the differential equation

$$
S_{y}^{2} C=\frac{1}{y^{2}} C
$$

Lemma A.4. The series $A(y)$ is a unique solution of equation (A.5).

Proof. Equation (A.5) implies that

$$
\left(\frac{\partial^{2}}{\partial y^{2}}+\left(\frac{4}{y}+\frac{2 u^{2}}{y^{4}}\right) \frac{\partial}{\partial y}+\frac{5}{4 y^{2}}\right) C=0 .
$$

The fact, that the series $A(y)$ is a unique solution of (A.6), can be checked by a simple direct computation.

Lemma A.5. The series $D$ satisfies the differential equation $\widehat{S}_{y} D=\frac{A(-y)}{y}$.

Proof. From (1.9) it immediately follows that for any $n \geq 1$ we have

$$
d_{n}=\left(3 n-\frac{3}{2}\right) d_{n-1}+\left|a_{n}\right|
$$

This easily implies the statement of the lemma.

Recall that the series $B(y)$ is defined by $B(y):=y S_{y} A(y)$.

Lemma A.6. We have $S_{y}\left(\frac{B(y)}{y}\right)=\frac{A(y)}{y^{2}}$.

Proof. The equation is equivalent to the equation $S_{y}^{2} A(y)=\frac{A(y)}{y^{2}}$, that follows from Lemma A.4.

Lemma A.7. For any $n \geq 0$ we have

$$
S_{y}^{n} A(y)=Q_{n} A(y)+R_{n} \frac{B(y)}{y}
$$

for some Laurent polynomials $Q_{n}, R_{n} \in \mathbb{C}\left[u^{-1}, y^{-1}\right]$, that are even as functions of $y$.

Proof. Define a sequence of Laurent polynomials $Q_{n}, R_{n} \in \mathbb{C}\left[u^{-1}, y, y^{-1}\right], n \geq 0$, by

$$
\begin{array}{ll}
Q_{0}=1, & R_{0}=0, \\
Q_{n+1}=\frac{y^{3}}{u^{2}} \frac{\partial Q_{n}}{\partial y}+\frac{1}{y^{2}} R_{n}, & R_{n+1}=Q_{n}+\frac{y^{3}}{u^{2}} \frac{\partial R_{n}}{\partial y} .
\end{array}
$$

This recursion immediately implies that $Q_{n}$ and $R_{n}$ are even as functions of $y$. Using this fact it is easy to see that, actually, $Q_{n}, R_{n} \in \mathbb{C}\left[u^{-1}, y^{-1}\right]$. Let us check that

$$
S_{y}^{n} A(y)=Q_{n} A(y)+R_{n} \frac{B(y)}{y} .
$$

The proof is by induction on $n$. This equation is obviously true for $n=0$. For an arbitrary $n$ we compute

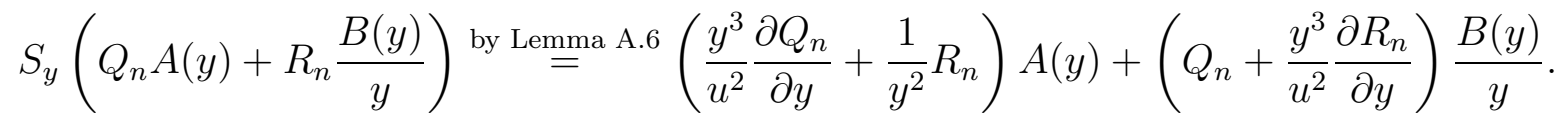

The lemma is proved. 


\section{REFERENCES}

[Ale14a] A. Alexandrov. Open intersection numbers, matrix models and MKP hierarchy. arXiv:1410.1820.

[Ale14b] A. Alexandrov. Open intersection numbers, Kontsevich-Penner model and cut-and-join operators. arXiv:1412.3772.

[BY14] M. Bertola, D. Yang. The partition function of the extended $r$-reduced Kadomtsev-Petviashvili hierarchy. arXiv:1411.5717.

[Bur14] A. Buryak. Equivalence of the open KdV and the open Virasoro equations for the moduli space of Riemann surfaces with boundary. arXiv:1409.3888.

[BT15] A. Buryak, R. J. Tessler. Matrix models and a proof of the open analog of Witten's conjecture. arXiv:1501.07888.

[BH15] E. Brezin, S. Hikami. Random Matrix, Singularities and Open/Close Intersection Numbers. arXiv:1502.01416.

[CvdLS14] G. Carlet, J. van de Leur, H. Posthuma, S. Shadrin. Towards Lax Formulation of Integrable Hierarchies of Topological Type. Communications in Mathematical Physics 326 (2014), no. 3, 815-849.

[DM69] P. Deligne, D. Mumford. The irreducibility of the space of curves of given genus. Publications mathématiques de l'I.H.É.S. 36 (1969), 75-109.

[Dic03] L. A. Dickey. Soliton equations and Hamiltonian systems. Second edition. Advanced Series in Mathematical Physics, 26. World Scientific Publishing Co., Inc., River Edge, NJ, 2003. xii+408 pp.

[DVV91] R. Dijkgraaf, H. Verlinde, E. Verlinde. Loop equations and Virasoro constraints in non-perturbative two-dimensional quantum gravity. Nuclear Physics B 348 (1991), no. 3, 435-456.

[KS91] V. Kac, A. Schwarz. Geometric interpretation of the partition function of 2D gravity. Physics Letters B 257 (1991), no. 3-4, 329-334.

[Kon92] M. Kontsevich. Intersection Theory on the Moduli Space of Curves and the Matrix Airy Function. Communications in Mathematical Physics 147 (1992), 1-23.

[PPZ13] R. Pandharipande, A. Pixton, D. Zvonkine. Relations on $\overline{\mathcal{M}}_{g, n}$ via 3 -spin structures. Journal of the American Mathematical Society 28 (2015), no. 1, 279-309.

[PST14] R. Pandharipande, J. P. Solomon, R. J. Tessler. Intersection theory on moduli of disks, open KdV and Virasoro. arXiv:1409.2191.

[ST] J. P. Solomon and R. J. Tessler, in preparation.

[Wit91] E. Witten. Two-dimensional gravity and intersection theory on moduli space. Surveys in differential geometry (Cambridge, MA, 1990), 243-310, Lehigh Univ., Bethlehem, PA, 1991.

A. BURYAK:

Department of Mathematics, ETH Zurich,

Ramistrasse 101 8092, HG G 27.1, Zurich, Switzerland.

E-mail address: buryaksh@gmail.com 